\title{
Electronic Speckle Pattern Interferometry with Optimum Image Extraction for Deformation Measurement under Environmental Disturbance*
}

\author{
Shuichi ARIKAWA**, Yusuke NAKAYA** and Satoru YONEYAMA** \\ ** Department of Mechanical Engineering, Aoyama Gakuin University \\ 5-10-1 Fuchinobe, Sagamihara, Kanagawa 252-5258, Japan \\ E-mail: arikawa@me.aoyama.ne.jp
}

\begin{abstract}
A method for extracting optimum images from a large amount of speckle images is proposed for ESPI measurement under environmental disturbance. During the measurement of static deformation under environmental disturbance, optimum images which can make interference fringes are extracted from the speckle images before the image subtraction. The extraction is performed based on the evaluation of the highest speckle contrast in time series value. The validity of the method is investigated by experiments under the environmental disturbance coming from the floor. Results indicate that the extraction method is effective and it is possible to measure static surface deformation by ESPI without a vibration isolator under environmental disturbance.
\end{abstract}

Key words: Deformation Measurement, Electronic Speckle Pattern Interferometry, Environmental Disturbance, Image Extraction, Speckle Contrast

\section{Introduction}

Electronic speckle pattern interferometry (ESPI) ${ }^{(1),(2)}$ is a very sensitive and noncontact method for the measurement of surface deformation distribution. The basics of this method is the image subtraction of an initial state from a deformed state image of the speckle pattern. ESPI has been applied to static/dynamic deformation measurement ${ }^{(3)-(9)}$, vibration mode analysis ${ }^{(10),(11)}$, non-destructive testing ${ }^{(12)-(14)}$ and shape measurement ${ }^{(15)}$. However, due to the sensitiveness of ESPI, it is difficult to use this method without a vibration isolator in the presence of the environmental disturbance coming from the ground or the floor. Moreover, because the environmental disturbance is random, the synchronous image recording with the vibration phase is also difficult. These difficulties impede applications of ESPI measurements to various fields, such as a pressure vessel in a power plant. As a solution to this problem, it can be considered that the application of high-speed or dynamic ESPI $^{(6)-(9)}$ which uses a high-speed camera and a high-power laser is available. In this situation, the state of rest of the object is not required, whereas it is required that the exposure time is short enough to freeze the object. However, the introduction of high-power laser to gain the sufficient intensity during the exposure requires relatively high costs.

On the other hand, in the measurement of static deformation under the environmental disturbance, there is a possibility that the static deformation is measured by ESPI, even if the exposure time is not so short. The reason for this is as follows. If a large number images of an initial and a deformed state are captured, naturally, it is considered that an optimum image set of the initial and the deformed state which can make interference fringes is included in them. In this situation, we can use an ordinary camera and an ordinary laser for

*Received 17 Nov., 2011 (No. 11-0698) [DOI: 10.1299/jmmp.6.634]

Copyright (C) 2012 by JSME 


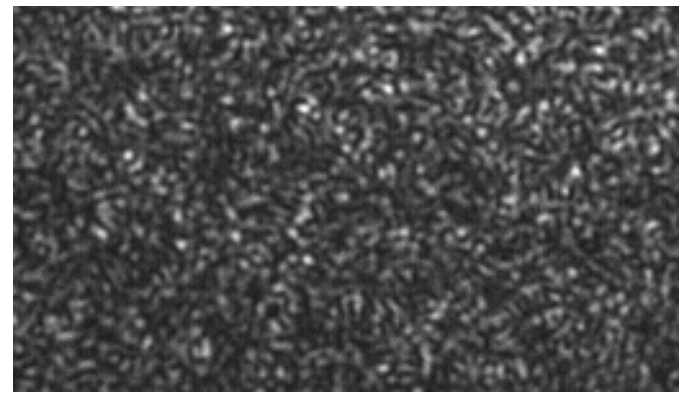

Fig. 1 Typical speckle pattern

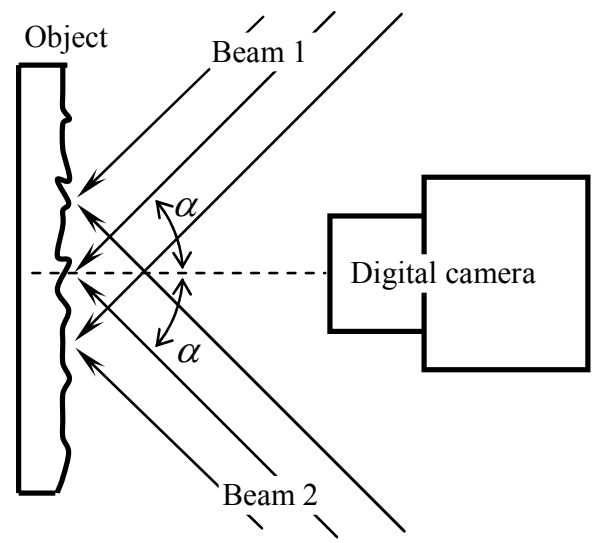

Fig. 2 Schematic figure of the dual-beam interferometer

the measurement without a vibration isolator. However, the calculation of all patterns of these image sets is not realistic because of the large quantity of data and the calculation time. Therefore, the extraction of the optimum image set is important.

In this study, a method for extracting the optimum image before the image subtraction in ESPI measurement under the environmental disturbance is proposed. The extraction is performed based on the evaluation of the highest speckle contrast in time series values. For some pixels in each image, absolute deviations of the intensity around the time average are calculated. The sums of the values in each image are examined. Then, the largest sum may indicate the optimum image. The validity of the method is investigated by experiments under environmental disturbance coming from the floor. In addition, the effect of difference of the exposure time while capturing the images is also investigated. As a result, it is verified that the extraction method is effective. Therefore, it is possible to measure static surface deformation by ESPI without a vibration isolator under the environmental disturbance.

\section{Outline of Electronic Speckle Pattern Interferometry}

When a coherent laser beam illuminates a rough surface, the speckle pattern shown in Fig. 1 is formed on the image plane by the random interference of the reflected beams from the surface. This pattern is stable and tracks surface displacements, hence can be used as a noncontact marker. When the surface is illuminated by two beams, the intensities of each speckle are modulated because of the superposition of the beams. The superposed intensity $I$ is written as ${ }^{(2)}$

$$
I=A+B \cos \varphi
$$

where $A$ is the average intensity, $B$ is the modulation factor, and $\varphi$ is the phase which is 


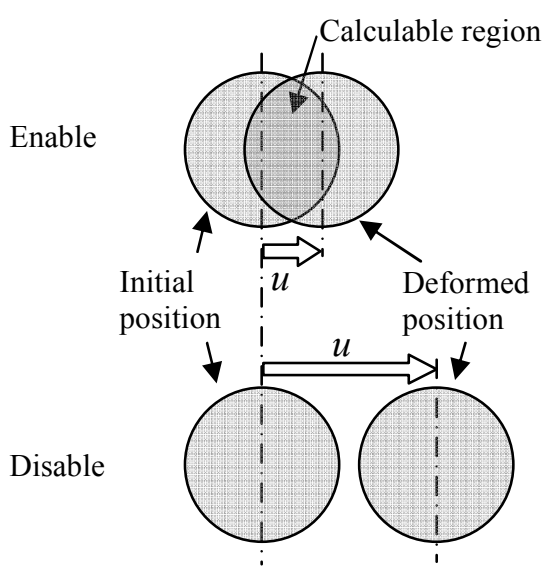

Fig. 3 Relationship between the speckle displacement and the speckle size

determined by the phase difference of the two beams at each position on the surface. A typical ESPI setup for in-plane displacement measuring is the dual-beam interferometer as shown in Fig. 2. The rough surface of the object is illuminated by the two beams i.e., the beam 1 and the beam 2 that are separated from a single light source. The interference fringe is given by the image subtraction of the deformed state from the initial state speckle pattern image. The intensity of the subtraction image $I_{\text {sub }}$ is calculated as ${ }^{(2)}$

$$
\begin{aligned}
I_{\text {sub }} & =\left|I_{\mathrm{u}}-I_{\mathrm{d}}\right|=\left|B\left\{\cos \varphi_{\mathrm{u}}-\cos \left(\varphi_{\mathrm{u}}+\Delta \varphi\right)\right\}\right| \\
& =\left|2 B \sin \left(\varphi_{\mathrm{u}}+\frac{\Delta \varphi}{2}\right) \sin \left(\frac{\Delta \varphi}{2}\right)\right|
\end{aligned}
$$

where $I_{\mathrm{u}}$ is the initial state intensity, $I_{\mathrm{d}}$ is the deformed state intensity, $\varphi_{\mathrm{u}}$ is the initial phase, and the $\Delta \varphi$ is the phase change of the deformed state from the initial state. The $\varphi_{\mathrm{u}}$ value of each point of the image is random and it causes the high frequency random noise as the random speckle pattern, but the $\Delta \varphi$ value makes the low frequency fringe pattern due to the surface deformation. When $\Delta \varphi / 2$ changes from zero to $2 n \pi$, the intensity of the fringe is modulated to $n$ cycles. The displacement due to the one cycle of the fringe intensity modulation, $u_{0}$ is given as

$$
u_{0}=\frac{\lambda}{2 \sin \alpha}
$$

where $\lambda$ is the wavelength of the laser beam and $\alpha$ is the incidence angle of the beams as shown in Fig. 2.

There are three major restrictions for producing the fringe pattern. The first restriction is the permissible gradient of the displacement. In other words, large strain is not permissible. A large strain makes the interference fringe pattern high frequency due to the term of $\sin (\Delta \varphi / 2)$ in Eq. (2). Therefore, the interference fringe cannot be identified. The second restriction is the permissible displacement. The image subtraction is performed on the same spatial coordinates of the initial and the deformed state image. If the displacement $u$ is increased, the calculable region cannot be obtained due to the relationship between the speckle size and $u$, as shown in Fig. 3. The average speckle diameter is determined by the formula of Airy disc. The diameter $d_{\mathrm{e}}$ of Airy disc is given as ${ }^{(2)}$

$$
d_{\mathrm{e}}=2.44 \lambda F
$$

where $F$ is the F-number of a camera lens determined by the value of the focal length divided by the lens diameter. However, the allowable displacement is smaller than $d_{\mathrm{e}}$, because it is affected by random noise and so on. The third restriction is the displacement 


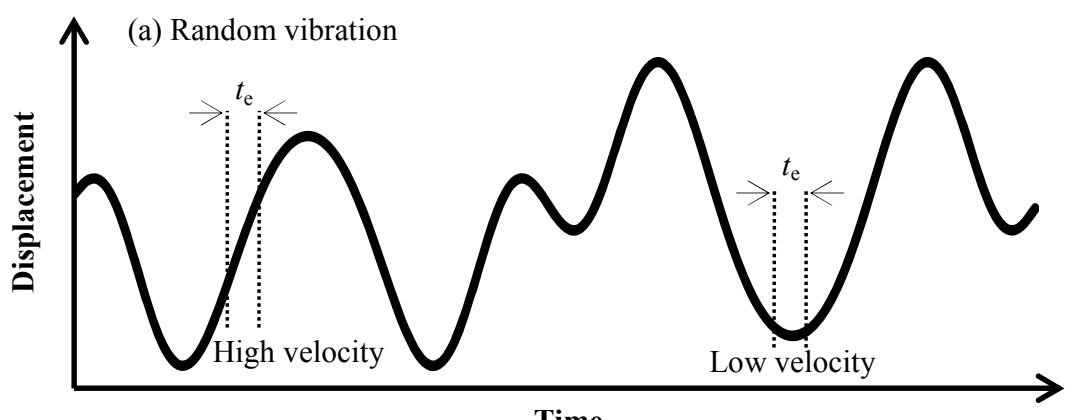

(b) High velocity

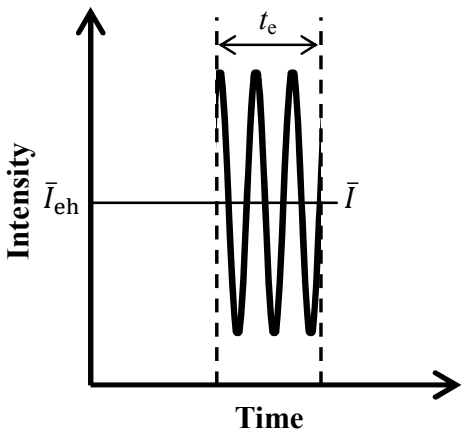

(c) Low velocity

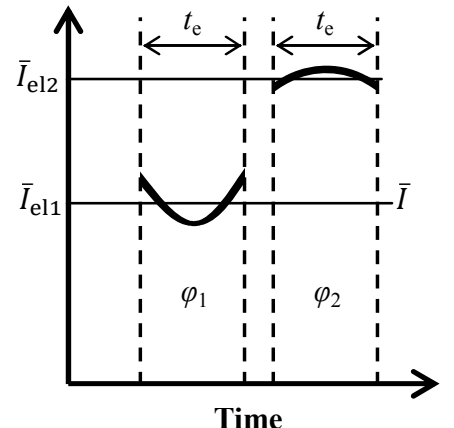

Fig. 4 Relationship between the displacement of the vibration and the speckle intensity: (a) a position-time profile of the vibration, (b) an intensity-time profile at high velocity and (c) at low velocity

during the exposure time, i.e., the object velocity. If the object velocity is too high, the modulation of the intensity becomes over one cycle. Because only the time averaged intensity can be recorded by the CCD during the exposure time, the term of $B \sin \varphi$ in Eq. (1) approaches zero, then the phase information disappears. In this paper, one solution to this problem is proposed.

\section{Method of Optimum Image Extraction}

Optimum image in this study means that the image is captured at a moment of sufficient low velocity of the object during the vibration. The proposed optimum image extraction method is based on the assumption that the dominant object motion caused by the disturbance is vertical translation. The relationship between the disturbance as random vibration and the intensity of the speckle can be drawn as Fig. 4 . The displacement of the disturbance and the time profile can be schematically drawn as shown in Fig. 4(a). The intensity values captured during the exposure time $t_{\mathrm{e}}$ at the moments of various velocities are considered. In the high velocity moment in Fig. 4(b), there are many cycles of the intensity modulation during the exposure time. Only the mean intensity value is captured. Here, the intensity value is obtained as $\bar{I}_{\mathrm{eh}}$. In this situation, $\bar{I}_{\mathrm{eh}}$ is almost the same as the average intensity $\bar{I}$ which is the same as $A$ in the Eq. (1). The change in $\bar{I}_{\text {eh }}$ value is very little, even if the phase of the intensity modulation changes. In contrast, in the low velocity moment shown in Fig. 4(c), captured intensities change corresponding with each phase change because the modulation of the intensity is shorter than one cycle. For example, in the situation of the phase $\varphi_{1}$, the captured intensity $\bar{I}_{\mathrm{el} 1}$ is the same as $\bar{I}$. On the other hand, in the phase $\varphi_{2}$, the captured intensity $\bar{I}_{\mathrm{e} 2}$ is different from $\bar{I}$. Accordingly, when the velocity is sufficiently low, there is a good possibility that the captured intensity is different from the average intensity. Actually, the distinction of the velocity is impossible by the evaluation of the intensity value of a single position because the vibration and the values of $A, B$ and $\varphi$ 


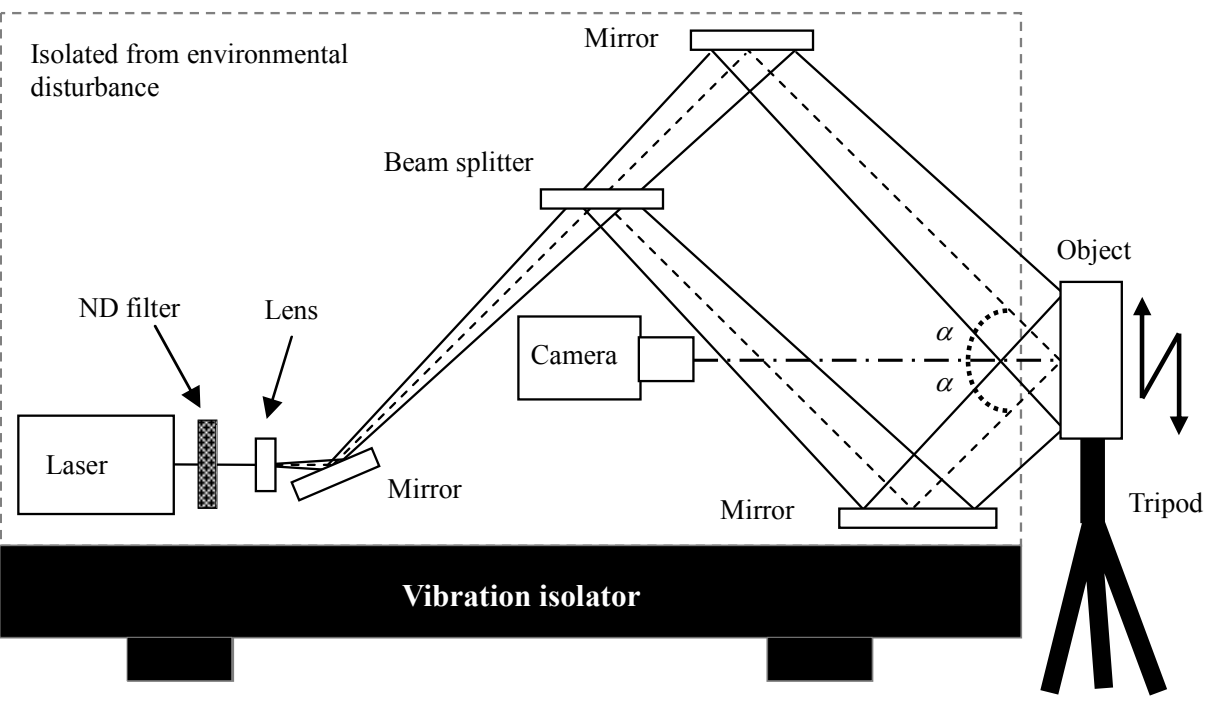

Fig. 5 Schematic figure of the dual-beam ESPI setup and the object

take random values at each spatial position on the image plane. Therefore, it is required that the evaluation of the difference between the intensity and the time average intensity value at the analyzing point is carried out for many positions.

The following method is proposed in this study. For some pixels in each image, the absolute deviations $e_{i j}$ of the intensity around the time average are calculated as

$$
e_{i j}=\left|I_{i j}-\bar{I}_{i}\right|
$$

where $i$ is pixel number of the image, $j$ is the image number of time series, $I_{i j}$ is the intensity value of the pixel number $i$ in the image number $j$, and $\bar{I}_{i}$ is the time average value at the pixel $i$. The sum $E_{j}$ of the values in each image is calculated as

$$
E_{j}=\sum_{i} e_{i j}
$$

Then, the largest sum $E_{\max }$ may indicate the optimum image.

\section{Experimental Setup and Procedure}

To investigate the validity of the proposed method, the experiments are performed under the environmental disturbance coming from the floor. The experimental setup used in this study is shown in Fig. 5. The ESPI setup is a typical dual-beam interferometer arranged to the vertical displacement measurement. A $75 \mathrm{~mW}$ He-Ne laser with the wavelength 632.8 nm (Melles Griot, model 05-LHP-928) is used. The CCD camera (SONY, model $\mathrm{XCD}-\mathrm{X} 710$ ) has a resolution of $1024 \times 768$ pixels, cell size of $4.65 \mu \mathrm{m} \times 4.65 \mu \mathrm{m}$ and maximum frame rate of 30 frames/s. The laser beam is separated by an unpolarizing beam splitter, and illuminates the object surface. The incidence angle of the beams is $45^{\circ}$, and the illumination region is about $70 \mathrm{~mm}$ in diameter. The ESPI setup is attached on a vibration isolator for the purpose of preventing relative fluctuation of the optics. On the other hand, the object attached on an ordinary camera stand is set on the floor. Therefore the object vibrates by the disturbance. A profile of the vibration is evaluated by measuring relative position between the interferometer and the object.

An interference fringe pattern caused by the in-plane rigid body rotation is observed. The rotation target is manually controlled. The images of the initial state and the rotated state of the object are captured. The frame rate for capturing the images is $10 \mathrm{frames} / \mathrm{s}$. In this study, it is expected that increasing exposure time will cause increasing displacement of vibration during exposure time. Therefore, in order to investigate the effect of the exposure 


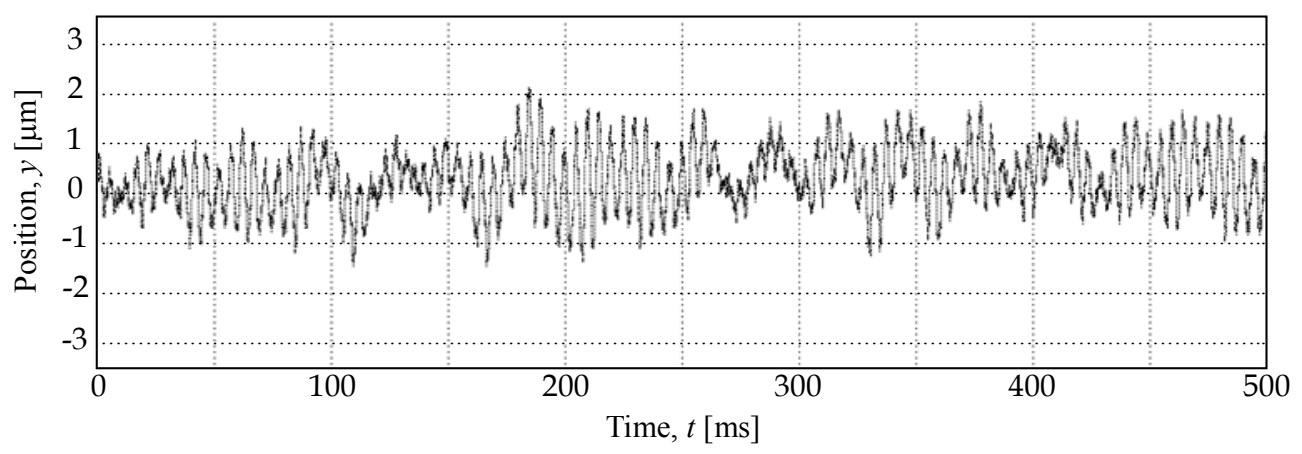

Fig. 6 Vibration profile of the relative position between the interferometer and the object

time on the optimum image extraction, the exposure time $t_{\mathrm{e}}$ is set to $1 / 250,1 / 100$ and $1 / 50 \mathrm{~s}$. In each setting of the exposure time, the laser power is adjusted by a ND filter to control the intensity. In this study, when the exposure time is $1 / 250 \mathrm{~s}$, the laser power is set to the maximum limit of $75 \mathrm{~mW}$. Therefore, when the exposure time is $1 / 100 \mathrm{~s}$ and $1 / 50 \mathrm{~s}$, the laser power corresponds to approximately $30 \mathrm{~mW}$ and $15 \mathrm{~mW}$ respectively by ND filters of transmittance of $40 \%$ and $20 \%$.

The experimental procedure is as follows. Before the rotation of the specimen, 100 images are captured. The evaluation method presented in this paper, Eqs. (5) and (6), are applied to each initial image. Then, intensity values at 100 points around the center in each image are evaluated. The image which shows $E_{\max }$ is extracted as the optimum image. In the same way, the $E_{\min }$ image which shows minimum $E_{j}$ value as the most unfit image is extracted for comparing with $E_{\max }$ image. Next, after the object rotation of about $0.004^{\circ}$ to counterclockwise, another 100 images are captured. Then, 100 subtraction images are calculated from the 100 rotated state images and the initial $E_{\max }$ image. In the same way, another 100 images are calculated from the initial $E_{\min }$ image. Then, the contrast levels and its frequency of the subtraction images are evaluated.

Additionally, a static strain measurement test is carried out for an aluminum sheet specimen by the strain-controlled tensile test. The dimension of the specimen is $20 \mathrm{~mm}$ in width, $50 \mu \mathrm{m}$ in thickness and about $100 \mathrm{~mm}$ in length. To verify the measurement accuracy, strain gage is attached on the specimen surface. The strain value that is measured by the proposed method is compared with the strain value that is measured by the strain gage.

\section{Results and Discussion}

\subsection{Profile of the disturbance coming from the floor}

Vibration as the disturbance coming from the floor is evaluated using an optical sensor probe (Yokogawa Electric Corporation). The relative position along the vertical axis between the interferometer and the object is measured by the probe fixed on the vibration isolator. The measurement result is shown in Fig. 6. As shown in the figure, amplitudes and frequencies are random. As a result of FFT analysis, the major frequency is about $20 \mathrm{~Hz}$. Therefore, it is considered that if the exposure time were longer than $1 / 20 \mathrm{~s}$, a possibility of capturing the optimum image would be very small. The maximum amplitude is about $2 \mu \mathrm{m}$. The distance of the maximum from the minimum position is about $3.5 \mu \mathrm{m}$. The distance causes decreasing contrast of interference fringes, but it does not extinguish the fringe contrast.

\subsection{Evaluation Value and Frequency}

Before the image subtraction, the extraction method is applied to the 100 initial state 


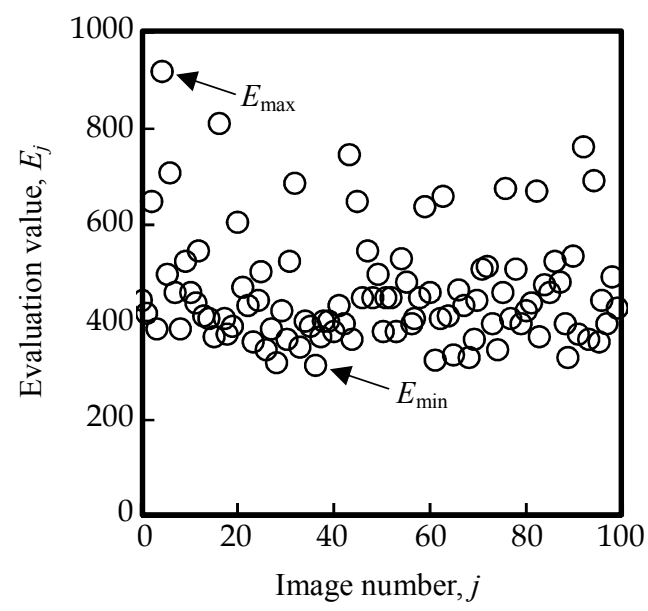

Fig. 7 Evaluation result of initial images
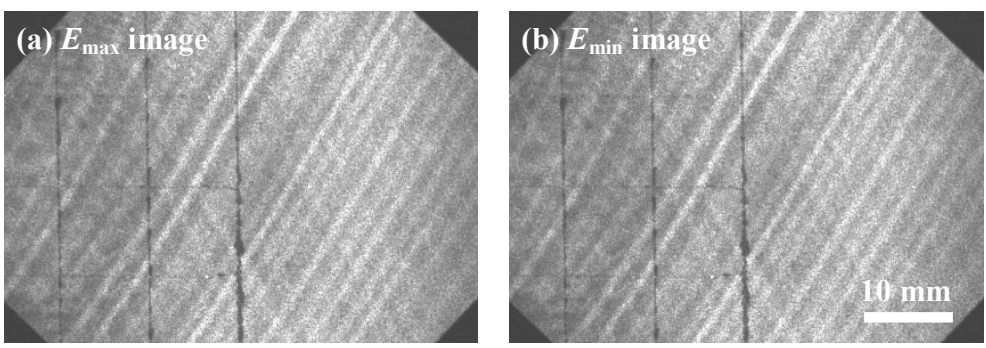

Fig. 8 Extracted initial speckle images of (a) $E_{\max }$ and (b) $E_{\min }$

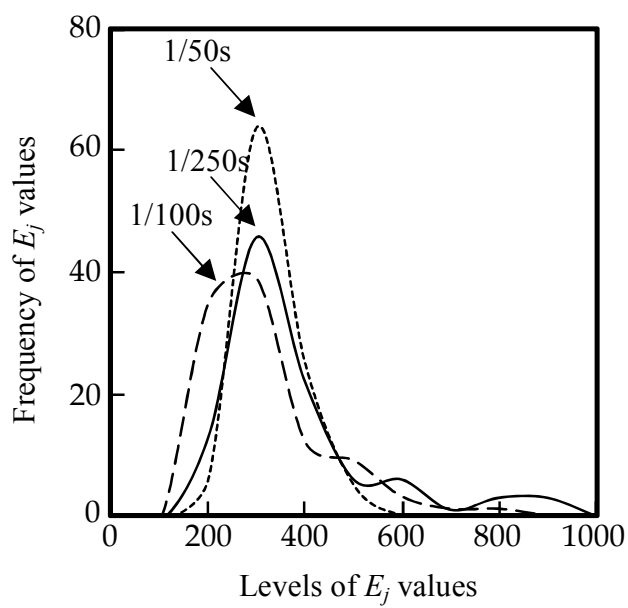

Fig. $9 E_{j}$ value frequency profiles of each exposure time

speckle images. Figure 7 shows the $E_{j}$ values as the evaluation result of the time series images. It is observed that the distribution of the $E_{j}$ value concentrates at around the value of 400 . The concentration decreases as the $E_{j}$ value increases. On the other hand, no value is observed in the lower $E_{j}$. The $E_{\max }$ and the $E_{\min }$ values are obtained as shown by the arrows in the Fig. 7. It is expected that dark current noise is slightly included in this result. Two images which indicate the $E_{\max }$ and the $E_{\min }$ are shown in Fig. 8. It is considered that the $E_{\max }$ image is the optimum image to make the interference fringe, and the $E_{\min }$ image is the most unfit one. However, the two images cannot be distinguished by the naked eye.

The frequencies of 100 intervals of the $E_{j}$ values are evaluated for each of the three exposure times, 1/250, 1/100 and 1/50 s. This has been represented in Fig. 9. Here, the 

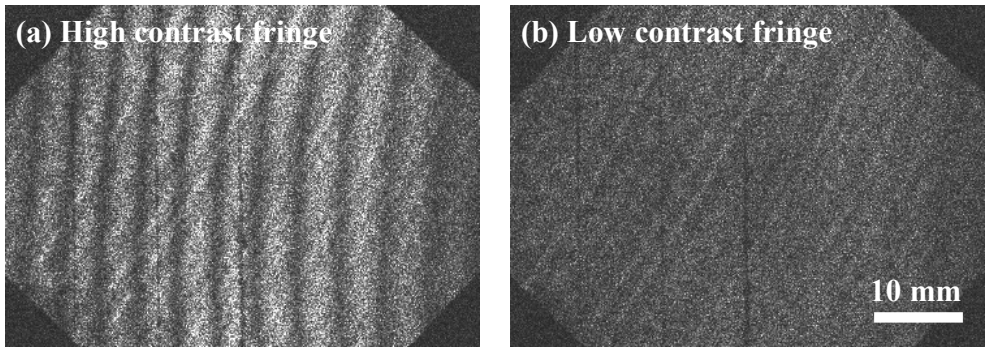

Fig. 10 Examples of subtraction images of (a) the high contrast fringes and (b) the low contrast fringes

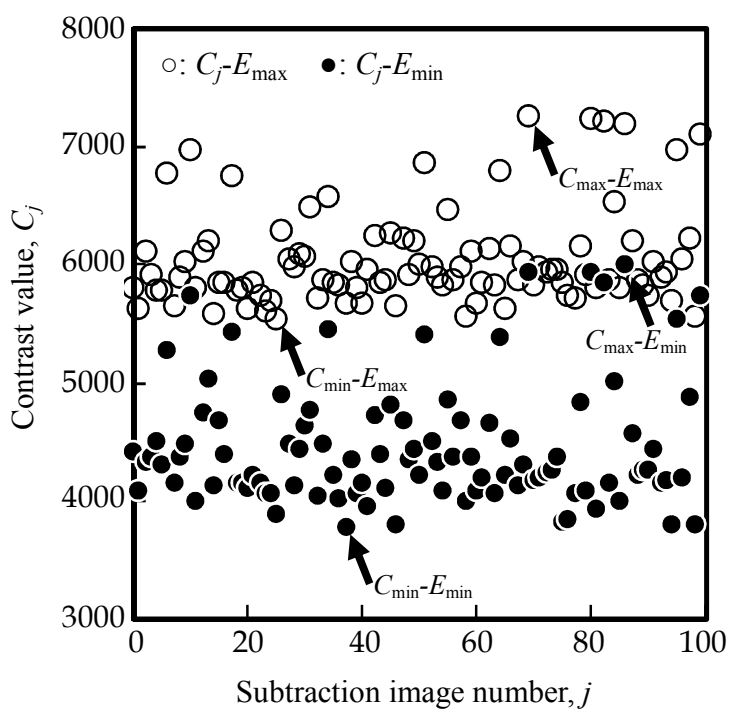

Fig. 11 Contrast evaluation result of subtraction images of the initial $E_{\max }$ image from deformed state images $\left(C_{j}-E_{\max }\right)$ and the initial $E_{\min }$ image from deformed state images $\left(C_{j}-E_{\min }\right)$

ordinate is the counts of the images and the abscissa is the range of the $E_{j}$ values. It is observed that the range of the highest frequency agrees with each condition. However, the highest $E_{j}$ value increases with the decrease of the exposure time.

\subsection{Contrast of the Interference Fringe and the Frequency}

The subtraction images are obtained from the $E_{\max }$ image and 100 images of the rotated state, also using the $E_{\min }$ image and the 100 images. The examples of the interference fringes of the high and low contrast images are shown in Fig. 10. Distance of the interference fringes is similar to expected distance from rotation angle. In this situation, the presence or absence of the interference fringes is easily judged. However, there are many other images the judgment of which is difficult. Therefore, the contrasts of these fringe images are evaluated. The contrast evaluation is carried out in a similar way as the optimum image extraction. The difference between the two is that the contrast evaluation uses the spatial average as oppose to the time average. The contrast evaluation is carried out for a series data of horizontal 100 points around the center of each image. The contrast values $C_{j}$ for the exposure time of $1 / 100 \mathrm{~s}$ is plotted as shown in Fig. 11. The open circles are the $C_{j}$ values obtained from the $E_{\max }$ image, $C_{j}-E_{\max }$ and the solid circles are the values obtained from the $E_{\min }$ image, $C_{j}-E_{\min }$. It can be observed that these distributions are clearly separated, as the $C_{j}-E_{\max }$ values concentrates at around 5800 and the $C_{j}-E_{\min }$ values concentrates at around 4200 . Also, the $C_{j}-E_{\min }$ value does not appear in the range up to about 6000 

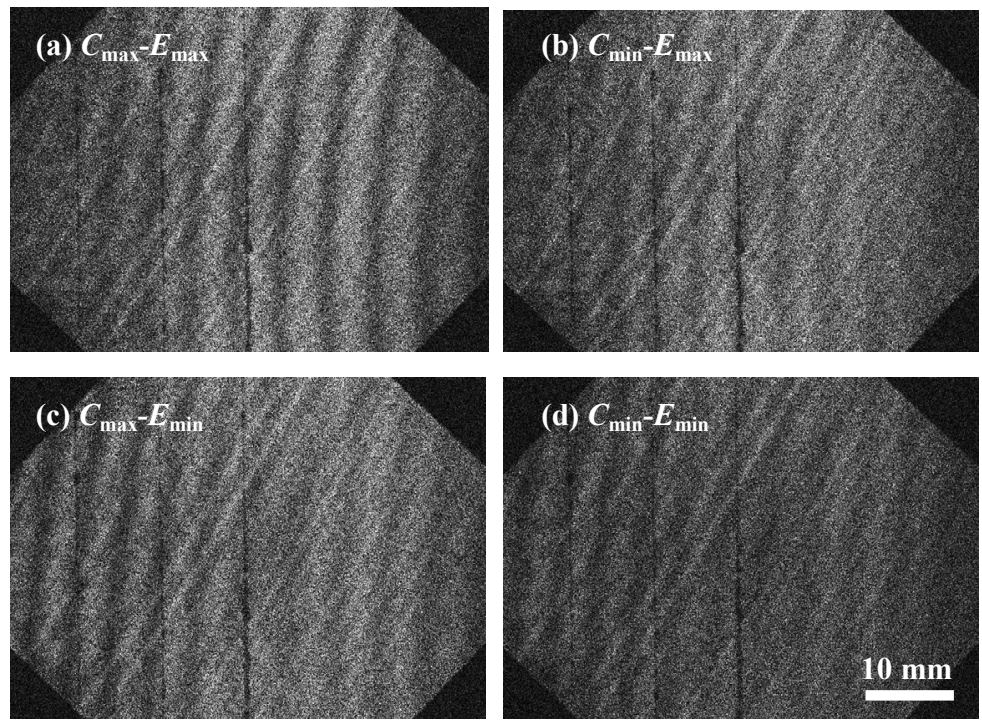

Fig. 12 Extracted images of highest and lowest fringe contrast calculated from $E_{\max }$ images: (a) $C_{\max }-E_{\max }$; (b) $C_{\min }-E_{\max }$, and calculated from $E_{\min }$ images: (c) $C_{\max }-E_{\min }$; (d) $C_{\min }-E_{\min }$

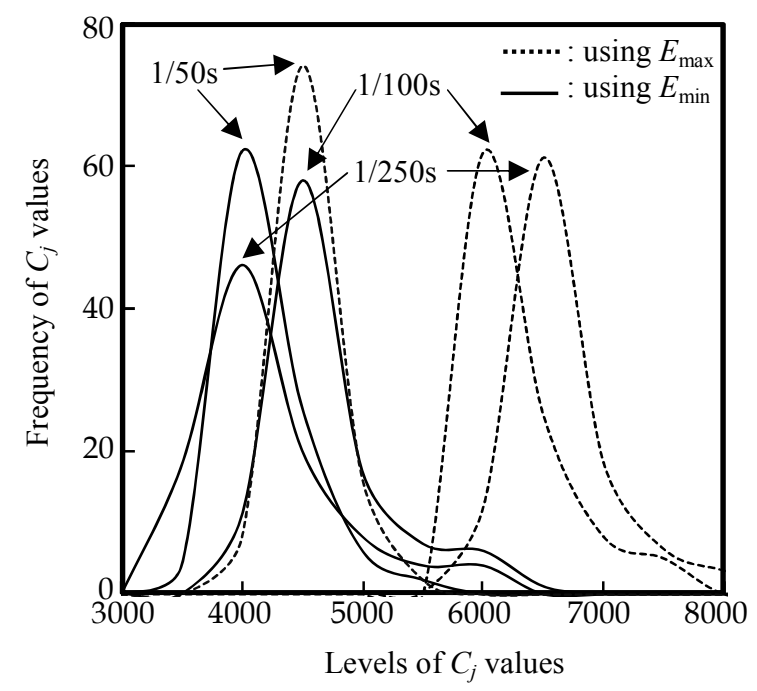

Fig. $13 C_{j}$ value frequency profiles of each exposure time

The actual subtraction images are shown in Fig. 12. Figure 12(a) is the image obtained from the maximum contrast of the $C_{j}-E_{\max }$, Fig. 12(b) is the image obtained from the minimum of the $C_{j}-E_{\max }$, Fig. 12(c) is the image obtained from the maximum of the $C_{j}-E_{\min }$ and Fig. 12(d) is the image obtained from the minimum of the $C_{j}-E_{\min }$. These values of the images are shown by the arrows in the Fig. 11. In particular, the image obtained from the minimum contrast of the $C_{j}-E_{\min }$ is mostly dark. The reason for this is that the images of two states are almost averaged, as the term $B \cos \varphi$ in Eq. (1) approaches zero, i.e., the subtraction result may approximate zero. The $C_{j}$ values shown in each image agree with contrast levels around center of images as can be seen by naked eye. Therefore, it is considered that the contrast evaluation is effective.

The frequencies of 100 intervals of $C_{j}$ values are evaluated for each of the three exposure times, 1/250, 1/100 and 1/50 s. This has been presented in Fig. 13. Here, the ordinate is the counts of the images and the abscissa is the range of the $C_{j}$ values. It is observed that positions of peaks of the $C_{j}$ values obtained from the images of $C_{j}-E_{\min }$ agree 


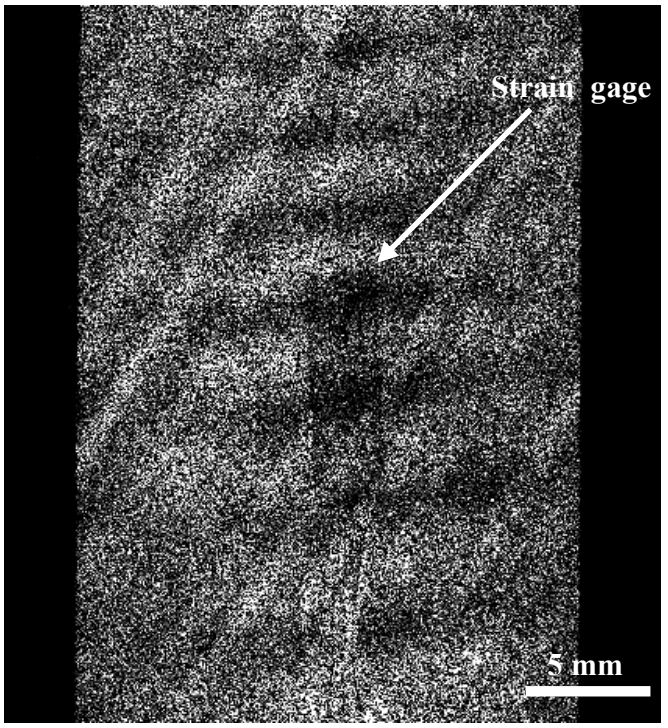

Fig. 14 Fringe pattern under tension

with each other and are observed at a level around 4000 to 4500 . On the other hand, positions of peaks of $C_{j}$ values obtained from the images of $C_{j}-E_{\max }$ disagree with each other. The peak for the exposure time $1 / 50 \mathrm{~s}$ is observed at a level of about 4500 . In contrast, the peaks for the exposure times $1 / 250 \mathrm{~s}$ and $1 / 100 \mathrm{~s}$ are increased as the decrease of the exposure time. The skirts of the distributions broaden with decreasing the exposure time. In particular, the distributions of the frequencies extend to higher $C_{j}$ level. The level of the $C_{j}$ value of almost all the $C_{j}-E_{\max }$ images for $1 / 250 \mathrm{~s}$ is not less than 6000 which is the value where the interference fringe can be easily observed by naked eye. In contrast, almost all the $C_{j}-E_{\max }$ images for $1 / 50 \mathrm{~s}$ is less than 5000 . However, capturing a large number of images over a long period of time will ensure that such optimum images would also be included in them, because the disturbance is random.

\subsection{Strain Measurement Test}

The interference fringe image obtained from the optimum image which is extracted by the proposed method is shown in Fig. 14. The interference fringes by the deformation appear along horizontal direction because the direction of the tensile test and the measurement sensitivity direction of the ESPI setup are vertical. Here, the slanting fringes appear by scratches of a mirror and hence has no relation to the measurement. In this study, phase analysis of the interference fringes is still not applicable and the image is quite noisy, but the peak distance of the fringes can be detected. The strain calculated from the distance of the horizontal fringes around the strain gage is $112 \times 10^{-6}$, whereas the strain measured by the strain gage is $106 \times 10^{-6}$. Both results have a good consistence. It is indicated that the proposed optimum image extraction method is effective for the static strain measurement in the presence of the environmental disturbance coming from the floor.

\section{Conclusion}

In this study, a method for extracting the optimum image before the image subtraction in ESPI measurement under environmental disturbance is proposed. The extraction is based on the evaluation of the highest speckle contrast. To investigate the validity of the method, the experiments are performed in the presence of the environmental disturbance coming from the floor. As a result, it is verified that the extraction method is effective. Therefore, it is possible to measure static surface deformation by ESPI without a vibration isolator under the environmental disturbance. 


\section{References}

(1) Chiang, F. P., Speckle Metrology, ASM handbook Volume 17, Nondestructive Evaluation and Quality Control (1989), p.432, ASM International.

(2) Sirohi, R. S., Speckle Interferometry, Contemporary Physics, Vol. 43, No. 3, (2002), pp. 161-180.

(3) Vial-Edwards, C., Lira, I., Martinez, A. and Münzenmayer, M, Electronic Speckle Pattern Interferometry Analysis of Tensile Test of Semihard Copper Sheets, Experimental Mechanics, Vol. 41, No. 1, (2001), pp. 58-62.

(4) Hinsch, K. D., Gülker, G. and Kelmers, H., Checkup for Aging Artwork - Optical Tools to Monitor Mechanical Behavior, Optics And Lasers in Engineering, Vol. 45, (2007), pp. 578588.

(5) Arikawa, S. and Yoneyama, S., A Simple Method for Detecting a Plastic Deformation Region Formed by Local Loading Using Electronic Speckle Pattern Interferometry (in Japanese), Transactions of the Japan Society of Mechanical Engineers, Series A, Vol. 77, No. 775, (2011), pp. 383-390.

(6) Madjarova, V., Toyooka, S., R, Widiastuti. and H, Kadono., Dynamic ESPI with Subtraction-Addition Method for Obtaining The Phase, Optics Communications, Vol. 212, (2002), pp. 35-43.

(7) Madjarova, V., Kadono, H. and Toyooka, S., Dynamic Electronic Speckle Pattern Interferometry (DESPI) Phase Analyses with Temporal Hilbert Transform, Optics Express, Vol. 11, (2003), pp. 617-623.

(8) Ikeda, T., Ichinose, K., Gomi, K. and Yoshida, S., Study of Dynamic Fracture Toughness Measuring Method by Electronic Spackle Pattern Interferometry (in Japanese), Proceedings of the Mechanical Engineering Congress, 2011Japan of the Japan Society of Mechanical Engineers, No. 7-1, (2007), pp. 57-58.

(9) Arikawa, S., Gaffney, J. A., Gomi, K., Ichinose, K., Ikeda, T., Mita, T., Rourks, R. L., Schneider, C. and Yoshida, S., Application of Electronic Speckle Pattern Interferometry to High-speed Phenomena, Journal of Material Testing Research Association of Japan, Vol. 52, No. 3, (2007), pp. 176-184.

(10) Ma, C. and Huang, C., Vibration Characteristics for Piezoelectric Cylinders Using Amplitude-Fluctuation Electronic Speckle Pattern Interferometry, AIAA Journal, Vol. 36, No. 12, (1998), pp. 2262-2268.

(11) Chen, C., Huang, C. and Chen, Y., Vibration Analysis and Measurement for Piezoceramic Rectangular Plates in Resonance, Journal of Sound and Vibration, No. 326, (2009), pp. 251 $-262$.

(12) Silva Gomes, J. F., Monteiro, J. M. and Vaz, M. A. P., NDI of Interface in Coating Systems Using Digital Interferometry, Mechanics of Materials, Vol. 32, (2000), pp. 837-843.

(13) Gryzagoridis, J., Findeis, D. and Tait, R. B., Residual Stress Determination and Defect Detection Using Electronic Speckle Pattern Interferometry, Insight - Non-Destructive Testing and Condition Monitoring, Vol. 47, No. 2, (2005), pp. 91-94.

(14) Ambu, R., Aymerich, F., Ginesu, F. and Priolo, O., Assessment of NDT Interferometric Techniques for Impact Damage Detection in Composite Laminates, Composites Science and Technology, Vol. 66, (2006), pp. 199-205.

(15) Parra-Michel, J., Martínez, A., Anguiano-Morales, A. and Rayas, J. A., Measuring Object Shape by Using In-plane Electronic Speckle Pattern Interferometry with Divergent Illumination, Measurement Science and Technology, Vol. 21, (2010), pp. 1-8. 